\title{
SIMULATION OF FREE SURFACE FLOWS BY A FINITE ELEMENT INTERFACE CAPTURING TECHNIQUE
}

\author{
L. Battaglia ${ }^{a, b * *}$, M. A. Storti ${ }^{a}$ and J. D'Elía ${ }^{a}$ \\ ${ }^{a}$ Centro Internacional de Métodos Computacionales en Ingeniería (CIMEC) \\ Instituto de Desarrollo Tecnologico para la Industria Química (INTEC) \\ UNL/CONICET \\ Güemes 3450, Santa Fe, Argentina \\ ${ }^{b}$ Grupo de Investigación en Métodos Numéricos en Ingeniería (GIMNI) \\ Facultad Regional Santa Fe, Universidad Tecnologica Nacional \\ Lavaisse 610, Santa Fe, Argentina
}

\begin{abstract}
.
Transient free surface flows are numerically simulated by a finite element interface capturing method based on a level set approach. The methodology consists of the solution of two-fluid viscous incompressible flows for a single domain, where the liquid phase is identified by positive values of the level set function, the gaseous phase by negative ones, and the free surface by the zero level set. The numerical solution at each time step is performed in three stages: (i) a two-fluid Navier-Stokes stage, (ii) an advection stage for the transport of the level set function, and (iii) a bounded reinitialization with continuous penalization stage for keeping smoothness of the level set function. The proposed procedure, and particularly the renormalization stage, are evaluated in three typical two and three-dimensional problems. Keywords: fluid mechanics, free surface, interface capturing, level set method, reinitialization.
\end{abstract}

\footnotetext{
${ }^{* *}$ Corresponding author. Email: lbattaglia@ $@$ santafe-conicet.gob.ar
} 


\section{INTRODUCTION}

Free Surface (FS) flows are a particular case of multiphase flows, where there are a liquid phase and a gaseous phase, the last one lighter than the first. Flows with a FS are found in several engineering disciplines, as chemical, mechanical or hydraulic, covering a wide range of fluid properties and flow cases, such as sloshing in liquid storage tanks or open channel flows. In this work, attention is focused on FS incompressible isothermal flows of Newtonian viscous fluids, in the cases where the surface tension is negligible.

There are different approaches for the computation of two-fluid flows, which are described as interface tracking and interface capturing methods (Shyy et al., 1996). On one hand, interface tracking methods follow explicitly the FS, which is defined over specific entities, such as nodes or faces of a mesh used with a Finite Element Method (FEM). The domain considers only the fluid phase, and the deformation of the domain, as a consequence of the FS movement, can be solved in different ways. The most common alternatives are Lagrangian approaches, as in particle methods (Idelsohn et al., 2004), where fluid particles are free to move, and Arbitrary Lagrangian-Eulerian (ALE) approaches (Hughes et al., 1981; Huerta and Liu, 1988; Chippada et al., 1996; Rabier and Medale, 2003), where the change in the shape of the domain involves either, the deformation of the mesh, keeping the topology constant, or a periodic remeshing, depending on the magnitude of the displacements. In the last alternative, large FS deformations are sometimes hard to model due to the fixed topology, avoiding merging or breaking up of the interface. On the other hand, interface capturing strategies consider fixed tessellations of the two-fluid domain, where the interface crosses a set of elements. The precise position of the FS is captured by an additional quantity, as a scalar field given over the whole domain, or a fluid fraction registered in each element crossed by the FS. Generally speaking, these alternatives allows the folding of the interface without special considerations, although they are not as precise as interface tracking regarding the FS displacements. The most common interface capturing methods are Volume of Fluid (VOF) (Hirt and Nichols, 1981; Scardovelli and Zaleski, 1999) and Level Set (LS) (Osher and Sethian, 1988; Sethian, 1995). The first one is based on the definition of a fluid fraction $F$ on each element of the discretization, being $F=0$ in the gaseous phase, $F=1$ in the liquid one and $0<F<1$ for cells crossed by the interface, which is recovered by specialized algorithms. On the other side, LS methods consider an additional scalar variable, the level set function $\phi$, which is advected by a transport equation, identifying the liquid phase with positive values $(\phi>0)$, the gaseous phase with negative values $(\phi<0)$ and the interface with null values $(\phi=0)$.

In previous works (Battaglia et al., 2006, 2007), a FEM-ALE strategy was developed for solving FS fluid flows such as sloshing in tanks. However, the proposal was limited to flow cases where the uniqueness of the interface was verified. The aim of this work is the application of a LS interface capturing approach for solving two-fluid flows in a single domain, considering both phases as Newtonian, viscous and incompressible fluids. In the present case, each fluid is indicated with a positive or negative value of the LS function $\phi$, as described before. The $\phi$-field is continuous over the whole domain, including the transition across the FS, given naturally at $\phi=0$.

The sequence of solution consist of three stages, which solve separately the following parts of the problem: (i) the fluid state, by solving the Navier-Stokes (NS) equations over the two-fluid domain; (ii) the transport of the LS function $\phi$, which implies the displacement of the interface $\phi=0$; and (iii) a reinitialization of the $\phi$-field for keeping the regularity of the LS function, which is specially important in the neighbourhood of the FS, and can be performed every certain 
number of time steps, according to the complexity of the flow (Battaglia, 2009). The numerical computation of each stage is made by different solvers of the PETSc-FEM libraries (PETScFEM, 2009), which are based on the Portable Extensible Toolkit for Scientific Computation (PETSc) libraries (Balay et al., 2008) and the Message Passing Interface (MPI, 2009) for parallel computing. The present results are obtained with an interface capturing approach that requires the synchronization among the three solvers involved and the development of a reinitialization stage.

The performance of the method is shown first, for the advection-renormalization of the LS field over a typical 2D example, and second, for two and three dimensional dam-break problems, which are compared to available experimental data (Martin and Moyce, 1952) and other numerical results.

\section{GOVERNING EQUATIONS}

The Partial Differential Equations (PDE) systems presented in this section are solved by FEM, and each one is related to each stage of solution. The NS and the advective (ADV) solvers have been previously analyzed (Sonzogni et al., 2002; Storti et al., 2008), while the so-called Bounded Renormalization with Continuous Penalization (BRCP) algorithm, which renormalizes the LS function field, was introduced only for advection-renormalization problems (Battaglia et al., 2010).

The multi-physics programming paradigm for the synchronization of the FEM modules was introduced in previous works (Battaglia et al., 2006). The three stages of solution run independently in parallel, and they are linked by $\mathrm{C}++$ synchronization programs named hooks, which are run at certain points in the FEM modules execution. The concept of the hooks has been borrowed from the GNU Emacs editor and from the Linux (2010) kernel. The hooks allow the data exchange among NS, ADV and BRCP solvers through the use of queues (first-in-first-out, FIFO). In each time step, the fluid velocities determined by the NS solver are sent to the ADV one for performing the LS function advection. Then, the LS field is sent to the BRCP module, where the renormalization is performed. After that, the NS stage receives the LS field, which is needed to determine the fluid properties in the whole domain for the following time step.

The time dependence is present in two of the three stages, the NS and the ADV, for which time integration is performed by the trapezoidal rule with parameter $\alpha$, being $\alpha=1$ for the Backward Euler method and $\alpha=0.5$ for Crank-Nicolson.

\subsection{Fluid state}

The fluid state in the domain $\Omega$ for time $t \in[0, T]$ is given by the NS equations system for two incompressible and immiscible fluids, which is:

$$
\begin{aligned}
\rho(\phi(\mathbf{x}, t))\left(\partial_{t} \mathbf{v}+\mathbf{v} \cdot \nabla \mathbf{v}-\mathbf{f}\right)-\nabla \cdot \boldsymbol{\sigma} & =0 ; \\
\nabla \cdot \mathbf{v} & =0 ;
\end{aligned}
$$

where $\mathbf{x} \in \Omega$ is the position vector, $\mathbf{v}$ is the fluid velocity, $\mathbf{f}$ is the body force by unit of mass, $\rho(\phi(\mathbf{x}, t))$ is the fluid density, $\partial_{t}(\ldots)=\partial(\ldots) / \partial t$ indicates the partial time derivative and $\phi$ is the LS function.

The fluid stress tensor $\boldsymbol{\sigma}$ is decomposed in an isotropic $-p \mathbf{I}$ part and a deviatoric one $\mathbf{T}$,

$$
\boldsymbol{\sigma}=-p \mathbf{I}+\mathbf{T}
$$


being $p$ the pressure, $\mathbf{I}$ the identity tensor and $\mathbf{T}$ the viscous forces tensor,

$$
\mathbf{T}=2 \mu(\phi(\mathbf{x}, t)) \boldsymbol{\epsilon}
$$

which is a function of the strain rate tensor $\epsilon$ determined as

$$
\boldsymbol{\epsilon}=\frac{1}{2}\left[\nabla \mathbf{v}+(\nabla \mathbf{v})^{T}\right]
$$

for Newtonian fluids, with $(\ldots)^{T}$ indicating transposition and being $\mu=\mu(\phi(\mathbf{x}, t))$ the dynamic viscosity.

The fluid properties, density and viscosity, depend on both, the position $\mathrm{x}$ and the evaluation time $t$ due to the multiphase model, which is given by the LS function $\phi$, defined over the whole domain $\Omega$. The values taken by $\phi$ indicate if the region $\Omega$ is occupied by one or another fluid (Sussman and Smereka, 1997), according to the following,

$$
\phi(\mathbf{x}, t)\left\{\begin{array}{lll}
>0 & \text { if } & \mathbf{x} \in \Omega_{l} \\
=0 & \text { if } & \mathbf{x} \in \Gamma_{\mathrm{FS}} \\
<0 & \text { if } & \mathbf{x} \in \Omega_{g}
\end{array}\right.
$$

where the subdomain $\Omega_{l}$ corresponds to the liquid phase and $\Omega_{g}$ is the gaseous one, while both conditions $\Omega=\Omega_{l} \cup \Omega_{g}$ and $\Omega_{l} \cap \Omega_{g}=\emptyset$ are verified. Note that the subindex adopted, $l$ and $g$, correspond to the liquid and the gaseous regions, respectively. Particularly, the FS is defined as

$$
\Gamma_{\mathrm{FS}}=\{\mathbf{x} \mid \phi(\mathbf{x}, t)=0\} .
$$

In this case, due to the renormalization method proposed for the LS function $\phi$, see Sec. 2.3, the function is bounded, i.e., $-1 \leq \phi \leq 1$, and the transition between fluids is smooth.

Given the LS value $\phi$, the fluid properties for Eqs. (1) and (4) are given as

$$
\begin{aligned}
& \rho(\phi)=\frac{1}{2}\left[(1+\tilde{H}(\phi)) \rho_{l}+(1-\tilde{H}(\phi)) \rho_{g}\right] \\
& \mu(\phi)=\frac{1}{2}\left[(1+\tilde{H}(\phi)) \mu_{l}+(1-\tilde{H}(\phi)) \mu_{g}\right]
\end{aligned}
$$

where the smeared Heaviside function $\tilde{H}(\phi)$ is determined through:

$$
\tilde{H}(\phi)=\tanh \left(\frac{\pi \phi}{\tilde{\varepsilon}}\right) ;
$$

where $\tilde{\varepsilon}$ is a reference parameter for the transition width. In this case, for $|\phi| \rightarrow \tilde{\varepsilon}, \tilde{H}(\phi) \rightarrow 1$, with the consequence of a diminishing in the width of the transition for the fluid properties needed for the NS system in comparison to the transition between $\phi=-1$ and $\phi=1$ considered in the ADV step. In particular, $\tilde{\varepsilon}=0.5$ is adopted, reducing the transition length in about $70 \%$.

The infinitely differentiable function $\tilde{H}(\phi)$ given in Eq. (8) is slightly different from other smooth Heaviside-like functions found in the literature (Sussman and Smereka, 1997; Olsson and Kreiss, 2005; Kurioka and Dowling, 2009) because it counts on a simpler mathematical expression, and it is not piecewise defined. This condition constitutes an advantage, because the selected function naturally fits the bounds $|\phi| \leq 1$ required by Eq. (7) for the interpolation of the fluid properties. 
For the fluid flow problems considered in this work, slip boundary conditions for the velocity $\mathbf{v}$ in Eq. (1) are given over the solid boundaries $\Gamma_{\text {wall, }}$, while in the case of the pressure, $p=0$ is imposed on top of the domain.

The solution of Eq. (1) is made through the NS solver from the PETSc-FEM libraries (Sonzogni et al., 2002), adopting linear elements with the same interpolation for velocity and pressure fields, which are stabilized with streamline upwind/Petrov-Galerkin (SUPG) (Brooks and Hughes, 1982) and pressure stabilizing/Petrov-Galerkin (PSPG) (Tezduyar et al., 1992).

\subsection{Level set function advection}

The transport of the LS function $\phi$ over the domain $\Omega$ is produced by the velocity $\mathbf{v}$ obtained by solving the NS equations, as follows,

$$
\partial_{t} \phi+\mathbf{v} \cdot \nabla \phi=0
$$

with boundary conditions given by

$$
\phi=\bar{\phi} \quad \text { over } \quad \Gamma_{\text {in }} ;
$$

where the inflow section is $\Gamma_{\text {in }}=\{\Gamma \mid \mathbf{v} \cdot \mathbf{n}<0\}$. In this way, the advection procedure takes into account the transport of the interface $\Gamma_{\mathrm{FS}}$ in a natural way.

This transport step, named ADV, is numerically solved by the advective module of the PETSc-FEM program (Storti et al., 2008). The numerical instabilities, which arise from the use of a Galerkin central scheme for solving the transport equation of the LS function $\phi$, can be avoided by a SUPG strategy (Brooks and Hughes, 1982). Nevertheless, if the BRCP is performed, the SUPG stabilization is not necessary for the transport of the LS function.

\subsection{Reinitialization of the level set function field}

Most LS approximations include a renormalization step, where a distance function equation is solved with the aim of keeping the regularity and the smoothness of the $\phi$-field; otherwise, the advection of $\phi$ or the interpolation of the fluid properties across the interface would lose precision in the numerical solution. Since some redistancing procedures are extremely expensive (Hysing, 2007), they are gradually replaced by improved algorithms (Elias et al., 2007; Mut et al., 2006). An alternative to the redistancing strategy consist of avoiding any reinitialization by performing a highly accurate transport of the LS field, such as discontinuous Galerkin methods (Marchandise and Remacle, 2006), high order Weighted Essentially Non-Oscillatory method, WENO (Kurioka and Dowling, 2009), or mesh adaptivity near $\phi=0$ (Marchandise and Remacle, 2006), among others.

In this work, the renormalization process is focused on conserving the regularity of the transition between phases by solving a PDE, introduced in Battaglia (2009). A similar strategy is the Conservative Level Set Method (Olsson and Kreiss, 2005; Olsson et al., 2007), although it does not count on a penalization term as in the present work.

The reinitialization consists of solving the PDE system by FEM, where the variable is $\phi$. The operator BRCP is

$$
\phi\left(\phi^{2}-\phi_{\mathrm{ref}}^{2}\right)-\kappa \Delta \phi+M\left(\hat{H}(\phi)-\hat{H}\left(\phi^{0}\right)\right)=0
$$

where $\kappa$ is a diffusive parameter, $M$ a penalty coefficient and $\phi_{\text {ref }}$ a reference value for the variable $\phi$, adopted as $\phi_{\text {ref }}=1$, while $\phi^{0}$ is the initial LS function value for the renormalization 


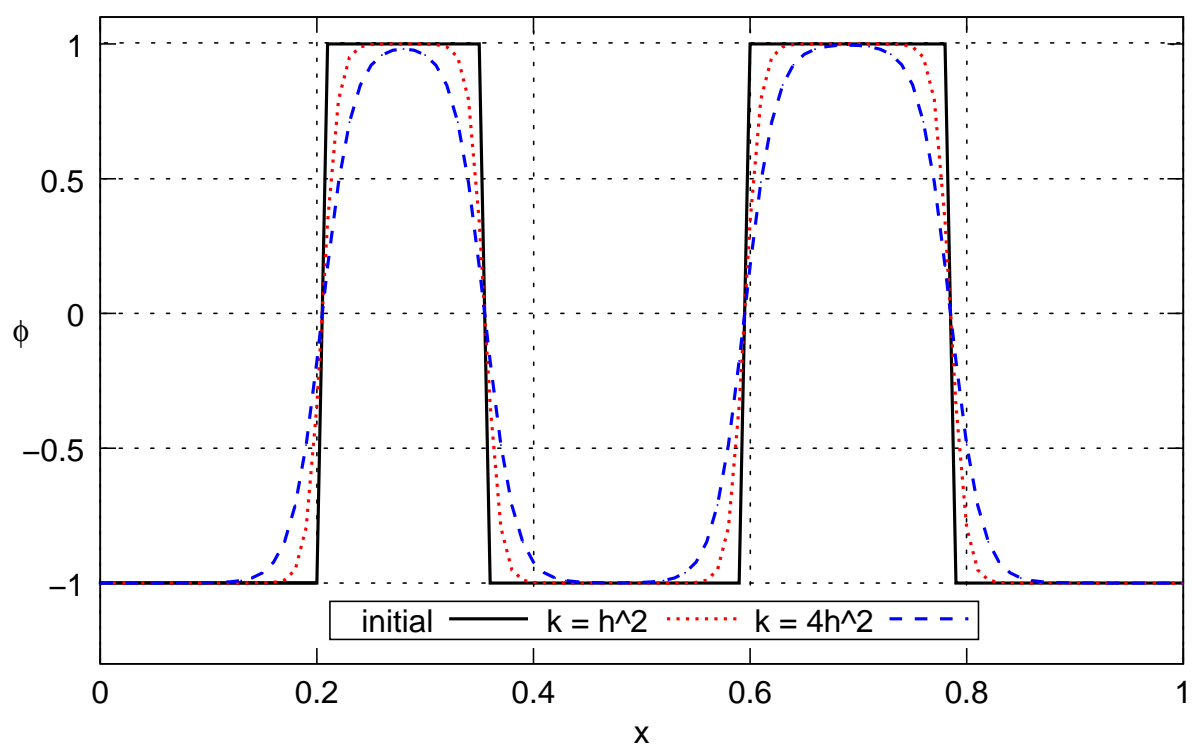

Figure 1: Artificial reaction-diffusion problem for renormalization of $\phi$ depending on the diffusion coefficient $\kappa$ value.

step provided by the solution of Eq. (9). The diffusion parameter $\kappa$, with squared length units, is related to an adopted typical element size $h$, usually from $h^{2}$ to $(3 h)^{2}$, depending on the FS behaviour, such that a lower $\kappa$ provides a thinner transition.

The positive coefficient $M$ is non-dimensional and should be adopted as $O\left(10^{n_{d}+2}\right)$, with $n_{d}$ the number of spatial dimensions involved. Furthermore, the function adopted for the penalizing term, $\hat{H}(\phi)$, has the continuous expression

$$
\hat{H}(\phi)=\tanh (2 \pi \phi)
$$

which is a smeared Heaviside function. The purpose of selecting a smooth function which is continuous across the interface is to avoid a complicated numerical treatment, especially in three dimensional problems.

The effect of Eq. (11) can be explained as follows. The first two terms constitute a steady heat conduction equation with a source term, being $\phi$ the temperature, i.e.

$$
\kappa \Delta \phi+Q(\phi)=0
$$

where $Q(\phi)=-\phi\left(\phi^{2}-\phi_{\text {ref }}^{2}\right)$ is the source term and $\kappa$ acts as a thermal diffusivity parameter.

Physically, the source term $Q(\phi)$ forces $\phi$ to reach the stable equilibrium temperatures, i.e. those $\phi^{*}$ for which $Q\left(\phi^{*}\right)=0$ and $Q^{\prime}\left(\phi^{*}\right)<0$, with $Q^{\prime}(\phi)=\mathrm{d} Q / \mathrm{d} \phi$. Since $Q\left(\phi^{*}\right)=0$, a spatially constant solution $\phi=\phi^{*}$ is a solution of Eq. (13). Therefore, if $\phi=\phi^{*}+\delta \phi$, with $\delta \phi$ a small perturbation, the source term is negative due to the condition $Q^{\prime}\left(\phi^{*}\right)<0$, and $\phi$ tends to $\phi^{*}$, independently of the sign of $\delta \phi$. For renormalization method, this condition is analogous for the roots $\phi^{*}= \pm \phi_{\text {ref }}$. Conversely, when $Q\left(\phi^{* *}\right)=0$ and $Q^{\prime}\left(\phi^{* *}\right)>0$, the roots $\phi^{* *}$ are unstable equilibrium temperatures, and any perturbation $\delta \phi$ produces a positive increment in the source term, taking $\phi$ away from the equilibrium value $\phi^{* *}$, as occurs when $\phi=0$ in Eq. (13).

Furthermore, the Laplacian term in Eq. (13) produces a smooth transition of semiwidth $\delta=$ $\mathcal{O}\left(\kappa^{1 / 2}\right)$ between the bounds given by the stable roots of the source term, which are $\phi^{*}= \pm \phi_{\text {ref }}$. Therefore, it is verified that a larger value of the diffusivity leads to a wider transition. This is shown in Fig. 1 for a steady problem with initial conditions given by piecewise-constants values for $\phi^{0}$, and the solutions of Eq. (13) corresponding to different values of diffusivity. 
When Eq. (11) is numerically solved over an unstructured or locally refined mesh, the diffusivity $\kappa$ should be chosen considering a given $\delta$ value, i.e. $\kappa=\mathcal{O}\left(\delta^{2}\right)$. An appropriate transition semiwidth $\delta$ can be given by the size of two or three elements near the interface $\phi=0$, regarding the precision of the numerical advection step.

The last term of Eq. (11), or penalizing term, takes into account the known values $\phi^{0}$, i.e. those determined in the advection step. The aim of this term is to avoid the displacement of $\phi=0$, i.e. the mass loss during the renormalization process, by weighting $\hat{H}(\phi)-\hat{H}\left(\phi^{0}\right)$. Convenient values for the penalty $M$ are $\mathcal{O}\left(10^{n_{\mathrm{d}}+2}\right)$, while low values such as $M=1$, lead to a higher error in the interface position, and with $M=0$ the algorithm fails because it lacks the reference $\phi^{0}$ from the advective step. For $M>\mathcal{O}\left(10^{n_{\mathrm{d}}+2}\right)$, the renormalization effect is lost, because the $\phi$-field tends to the $\phi^{0}$-field, and the solution given by the ADV step is recovered (Battaglia et al. (2010)). Penalty parameters for preserving the interface position were also proposed by other authors, as in the Edge-Tracked Interface Locator Technique (ETILT) method (Tezduyar (2006); Cruchaga et al. (2007)).

The renormalization process acts mainly in the neighbourhood of the FS, where the loss of precision in the FS position and the mass loss are registered (Mut et al., 2006; Cruchaga et al., 2007). On the other side, when $\phi \approx \phi_{\text {ref }}$, i.e. far from the interface, the three terms in Eq. (11) tend to zero. Finally, the operator of Eq. (11) is numerically solved by FEM on each time step or every $n_{\text {reno }}$ time steps, after the computation of the ADV stage.

\section{NUMERICAL EXAMPLES}

\subsection{Advection-renormalization problem: the disk of Zalesak}

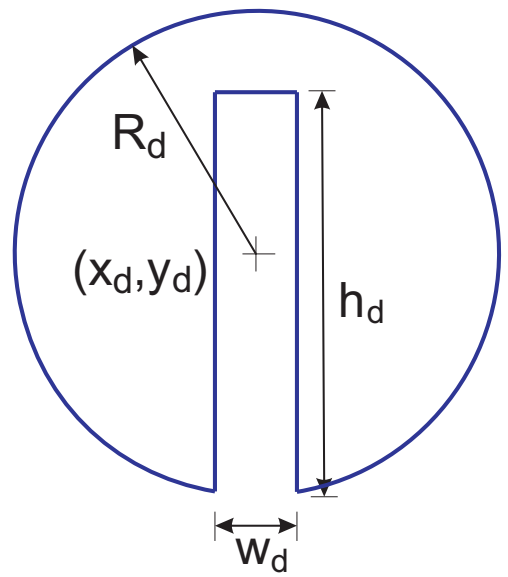

Figure 2: Shape of $\phi=0$ for the disk of the Zalesak test.

The effectiveness of the renormalization method is evaluated by solving the problem presented by Zalesak (1979) and reproduced by several authors (Elias and Coutinho, 2007; Gois et al., 2008; Mut et al., 2006), which consists of a notched disk inside a square unit domain with $0 \leq(x, y) \leq 1$. The initial condition are shown in Fig. 2, being the radius $R_{d}=0.15 \mathrm{~m}$, while the notch is $w_{d}=0.05 \mathrm{~m}$ wide and $h_{d}=0.25 \mathrm{~m}$ height. The disk is centered at $\left(x_{d}, y_{d}\right)=(0.5,0.75) \mathrm{m}$ at the beginning of the computation, Fig. 3, enclosing the $\phi>0$ region, and the velocity field is given by

$$
\begin{aligned}
& v_{x}=2 \pi\left(y-y_{c}\right) ; \\
& v_{y}=-2 \pi\left(x-x_{c}\right) ;
\end{aligned}
$$


which produces a rigid rotation of the notched disk around the point $\left(x_{c}, y_{c}\right)=(0.5,0.5) \mathrm{m}$. After one revolution, the numerical results are compared to the initial position, that should be recovered.

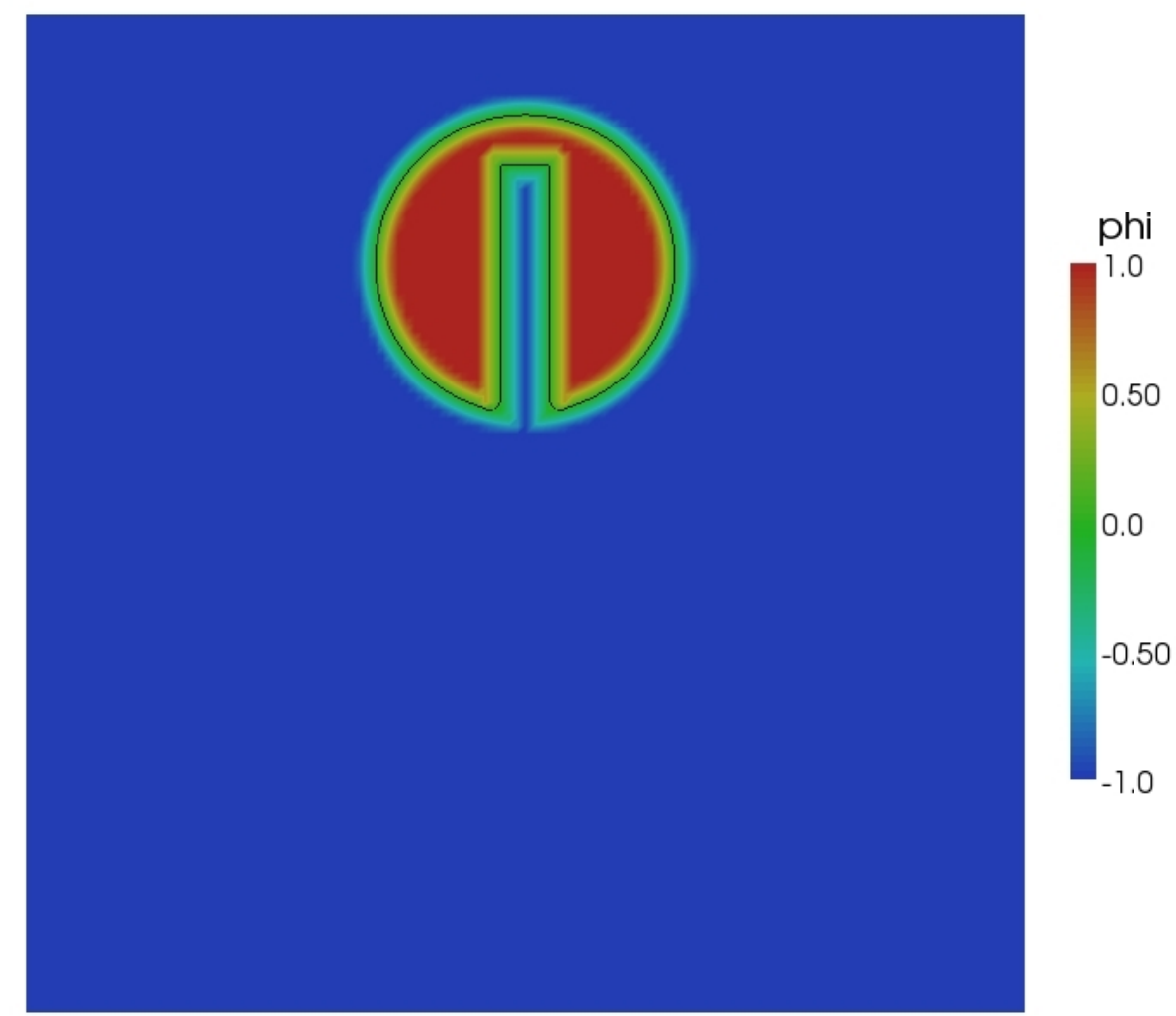

Figure 3: Initial level set function field for the notched disk for $h=7.8 \times 10^{-3} \mathrm{~m}$.

The problem is solved by two different strategies: using only Eq. (9), and with Eq. (9) plus the periodic renormalization with Eq. (11), named ADV and ADV+BRCP, respectively. For one revolution, the final time of $t_{f}=1 \mathrm{~s}$ is discretized in 628 time steps of $\Delta t=1 /(200 \pi) \mathrm{s} \approx$ $0.0016 \mathrm{~s}$, with an implicit time integration considering $\theta=0.7$ for the trapezoidal rule. The finite element mesh consists of $128^{2}$ bilinear quadrilateral elements of typical size $h=7.8 \times$ $10^{-3} \mathrm{~m}$.

The diffusivities for the renormalized examples are $\kappa_{\mathrm{A}}=2 h^{2}=1.22 \times 10^{-4} \mathrm{~m}^{2}$ in case $\mathrm{ADV}+\mathrm{BRCP}(\mathrm{A})$, or $\kappa_{\mathrm{B}}=4 h^{2}=2.44 \times 10^{-4} \mathrm{~m}^{2}$ in case $\mathrm{ADV}+\mathrm{BRCP}(\mathrm{B})$, while the penalty is chosen as $M=10000$ in both cases. The renormalization is performed every $n_{\text {reno }}=4$ advection time steps.

The performance of the three solution alternatives are shown in Fig. 4, where the profiles of $\phi=0$ in $t=1 \mathrm{~s}$ are represented for the three alternatives: (a) pure advection, (b) advection plus renormalization with $\kappa_{\mathrm{A}}=2 h^{2}$ and (c) advection plus renormalization with $\kappa_{\mathrm{B}}=4 h^{2}$. Notice that in the pure advection case the notch is almost disappeared, while in (b) it is well captured. Furthermore, the disappearance of the notch in (c) is attributed to the wider transition induced by a higher value of $\kappa$ than in (b), as explained in Sec. 2.3. The shape distortion of the disk in the results computed with $\mathrm{ADV}+\mathrm{BRCP}(\mathrm{A})$ is similar to the one presented in Kurioka and Dowling (2009) for the same problem with a $100^{2}$ elements mesh and a high order advection solver. 


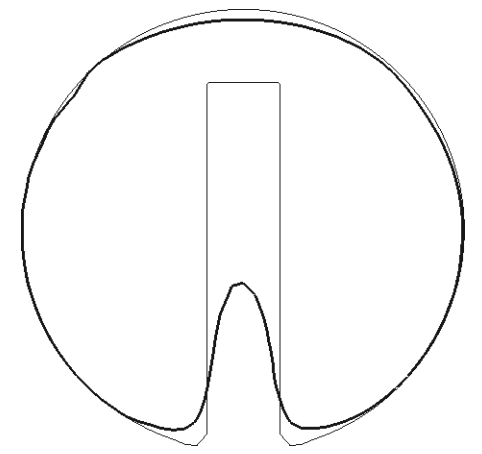

(a) $\mathrm{ADV}$

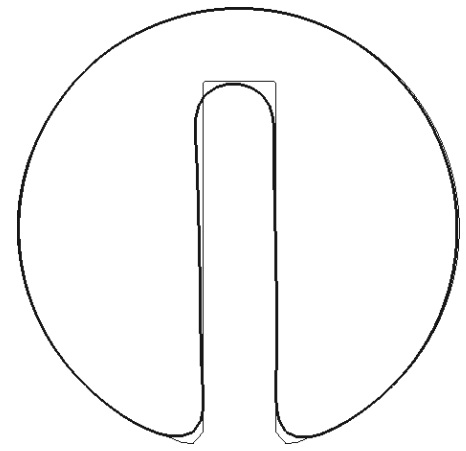

(b) $\mathrm{ADV}+\mathrm{BRCP}(\mathrm{A})$

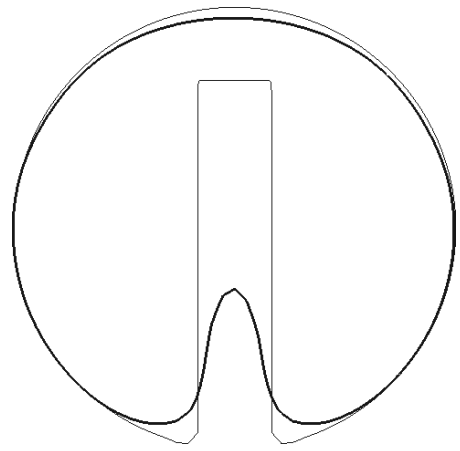

(c) $\mathrm{ADV}+\mathrm{BRCP}(\mathrm{B})$

Figure 4: Curve of $\phi=0$ for the disk of Zalesak at $t=1 \mathrm{~s}$ solved without and with BRCP (thick lines), together with the initial condition (thin lines).

Regarding area conservation of the $\phi>0$ region, there are few differences among the alternatives $\mathrm{ADV}$ and $\mathrm{ADV}+\mathrm{BRCP}(\mathrm{B})$, where the disk gains between 9 and $10 \%$ of area due to the disappearance of the notch, while in case $\mathrm{ADV}+\mathrm{BRCP}(\mathrm{A})$ the area decreases about $1 \%$.

Figure 5 shows the LS function field for (a) ADV and (b) ADV+BRCP(A). In the first case, the transition from -1 to +1 is not uniform, the relief is smoothed, and the notched disk tends to disappear. In case $\mathrm{ADV}+\mathrm{BRCP}$, the transition presents uniform width around the whole $\phi=0$ curve. Then, the constant width provided by the BRCP stage replaces the redistancing procedures classically performed in LS methods (Sussman and Smereka, 1997; Sussman et al., 1999).

Additionally, the same problem is solved with ADV+BRCP over a $256^{2}$ elements mesh with time step $\Delta t_{\mathrm{r}}=8 \times 10^{-4} \mathrm{~s}$, diffusivity $\kappa_{\mathrm{r}}=2 h_{\mathrm{r}}^{2}=3.05 \times 10^{-5} \mathrm{~m}^{2}$, penalty $M_{\mathrm{r}}=10000$ and $n_{\text {reno }}=4$. The LS function field and the final profile obtained with this refined mesh are shown in Fig. 6.

The difference in the width of the transitions in Figs. 6(a) and 5(b) is due to the diffusivity values chosen in each case, which are $\kappa_{\mathrm{r}}=3.05 \times 10^{-5} \mathrm{~m}^{2}$ and $\kappa_{\mathrm{A}}=1.22 \times 10^{-4} \mathrm{~m}^{2}$, respectively. Regarding the property $\delta=\mathcal{O}\left(\kappa^{1 / 2}\right)$, then $\delta_{\mathrm{r}} \approx 2 \kappa_{\mathrm{r}}^{1 / 2}=0.012 \mathrm{~m}$ for Fig. 6(a) and $\delta_{\mathrm{A}} \approx 2 \kappa_{\mathrm{A}}^{1 / 2}=0.022 \mathrm{~m}$ for Fig. 5(b). Furthermore, for the case $\operatorname{ADV}+\mathbf{B R C P}(\mathbf{B})$, $\kappa_{\mathrm{B}}=2.44 \times 10^{-4} \mathrm{~m}^{2}$ and $\delta_{\mathrm{B}} \approx 2 \kappa_{\mathrm{B}}^{1 / 2}=0.032 \mathrm{~m}>\mathrm{w}_{\mathrm{d}} / 2$, i.e. the proposed transition is too smooth and the method is not able to keep the notch width, as shown in Fig. 4(c).

\subsection{Collapse of a liquid column in 2D}

This example is a typical test for interface-capturing methods (Cruchaga et al., 2007; Elias and Coutinho, 2007; Kurioka and Dowling, 2009; Marchandise and Remacle, 2006; Tang et al., 2008), consisting of a two-dimensional water column which collapses after being liberated, and is also known as the dam-break problem. Experimental results are available for different geometrical configurations and fluids, e.g. see Martin and Moyce (1952); Cruchaga et al. (2007), allowing the validation of numerical methods.

The domain $\Omega$ considered for the numerical simulation is sketched in Fig. 7, being the domain of width and height $W_{d}=0.228 \mathrm{~m}$ and $H_{d}=0.228 \mathrm{~m}$, respectively, while the initial liquid domain $\Omega_{l}$-the water column- is $W_{c}=0.057 \mathrm{~m}$ width and $H_{c}=0.114 \mathrm{~m}$ height, resulting in an aspect ratio of $r_{a}=H_{c} / W_{c}=2$, the same as in the physical model of Martin and Moyce (1952).

The fluids considered for the simulation are air for the gaseous phase, with density $\rho_{g}=$ 
$1 \mathrm{~kg} / \mathrm{m}^{3}$ and dynamic viscosity $\mu_{g}=1.0 \times 10^{-5} \mathrm{~kg} /(\mathrm{m} \mathrm{s})$, and water for the liquid phase, being its density and dynamic viscosity $\rho_{l}=1000 \mathrm{~kg} / \mathrm{m}^{3}$ and $\mu_{l}=1.0 \times 10^{-3} \mathrm{~kg} /(\mathrm{m} \mathrm{s})$, respectively.

For the fluid problem, boundary conditions are a perfect slip over the whole contour, $\mathbf{v} \cdot \mathbf{n}=$ 0, as indicated in Fig. 7, being $\mathbf{n}$ the unit normal to the contour, and null pressure on the top of the domain. Boundary conditions for the transport problem are not required because there are no inflow sections for the domain $\Omega$.

The initial velocity field is $\mathbf{v}_{0}=\mathbf{0}$ for the NS and the ADV problems, while the initial LS function field is given such that the water column $\Omega_{l}$ includes nodes where $0<\phi \leq 1$, while in the rest of the domain $\Omega$, i.e. the gaseous phase $\Omega_{g}$, is $-1 \leq \phi<0$, and $\phi=0$ is the initial FS position. For the numerical simulation, the gate is instantaneously removed and the column collapses due to the gravity acceleration $g=9.81 \mathrm{~m} / \mathrm{s}^{2}$.

The numerical problem is solved for a uniform structured finite element mesh composed by quadrilaterals with typical size $h \approx 0.0023 \mathrm{~m}$ and approximately 10200 nodes, which is the same for the three stages to be solved: NS, ADV and BRCP. The time step adopted is $\Delta t=0.002 \mathrm{~s}$ along 1000 time steps, with an implicit temporal integration for the NS and the ADV problems, considering an integration parameter $\theta=0.7$ for the trapezoidal rule, while the BRCP stage is stationary each time step.

The reinitialization step is proposed with parameters $\kappa=2 h^{2}=1.04 \times 10^{-5} \mathrm{~m}^{2}$ and $M=$ 10000 . The number of ADV steps which are performed before each reinitialization is $n_{\text {reno }}=2$ for this particular example.

The dimensionless front position $x_{f}\left(t^{*}\right) / W_{c}$ is represented versus the dimensionless time $t^{*}=t \sqrt{2 g / W_{c}}$ in Fig. 8, for the numerical results and the experimental measurements taken from Martin and Moyce (1952). In that figure, the slope of the curve represents the velocity of the advancing front, which is well captured.

Other reference results, also represented in Fig. 8, were numerically obtained by using the ETILT method, from Cruchaga et al. (2007), and a VOF approach developed by Elias and Coutinho (2007). In both references, the aspect ratio is $r_{a}=H_{c} / W_{c}=2$, which means that there is a physical similarity of the problem solved with different $W_{c}$ values, according to the dimensionless front position and dimensionless time. Furthermore, Elias and Coutinho (2007) solved the collapse of the column in a 3D domain instead a two-dimensional one.

The evolution of the dimensionless water height $h_{c}\left(t^{*}\right) / W_{c}$, measured over the left side of the domain, is represented as a function of the dimensionless time $t^{*}=t \sqrt{2 g / W_{c}}$ in Fig. 9 . In that figure, the displacement of the numerical curve from the experimental one is smaller than in Fig. 8, and the mean descending velocity is well captured, considering the slope of the curves. Again, numerical reference results are taken from Cruchaga et al. (2007) and Elias and Coutinho (2007), that are also included in Fig. 9.

The FS position in different instants of the simulation are shown in Fig. 10, including early stages without breaking, as in $t=0.14 \mathrm{~s}$ or $t=0.28 \mathrm{~s}$, and later stages with air capture and interface merging.

\subsection{Collapse of a liquid column in 3D}

The three-dimensional example presented is the numerical simulation of the collapse of a cylindrical water column, which was also experimentally studied by Martin and Moyce (1952). The problem was solved for one fourth of the column inside a cubic domain, as represented in Fig. 11, with proper boundary conditions in order to keep the symmetry of the problem, as 
made by other authors (Akin et al., 2007; Cruchaga et al., 2009; Tang et al., 2008).

The domain $\Omega$ consists of a cube with edge length $b=0.2284 \mathrm{~m}$, in which the water column, i.e. the fluid domain $\Omega_{l}$, is centered in the corner of the plane coordinates $\left(x_{1}, x_{2}\right)=$ $(0.2284,0.2284) \mathrm{m}$, being its radius and height $r_{0}=0.0571 \mathrm{~m}$ and $h_{0}=0.1142 \mathrm{~m}$, respectively, giving an aspect ratio $r_{a}=2$, see Fig. 11 .

The collapse is started once the column is released instantaneously at time $t=0$ due to the action of the gravity acceleration $g=9.81 \mathrm{~m} / \mathrm{s}^{2}$, given in $-x_{3}$ direction. The fluid properties for water are density $\rho_{l}=1000 \mathrm{~kg} / \mathrm{m}^{3}$ and dynamic viscosity $\mu_{l}=1.0 \times 10^{-3} \mathrm{~kg} /(\mathrm{m} \mathrm{s})$, while for the air are $\rho_{g}=1 \mathrm{~kg} / \mathrm{m}^{3}$ and $\mu_{g}=1.0 \times 10^{-5} \mathrm{~kg} /(\mathrm{m} \mathrm{s})$.

The finite element mesh employed for the numerical simulation counts on $50^{3}$ hexahedral elements with uniform edge length $h \approx 4.5 \times 10^{-3} \mathrm{~m}$. Numerical simulation is performed along 1000 time steps of $\Delta t=0.001 \mathrm{~s}$ with implicit integration for NS and ADV, and renormalization at each time step, i.e. $n_{\text {reno }}=1$, adopting a penalizing parameter of $M=500000$ and a diffusion coefficient $\kappa=2 h^{2}=4.17 \times 10^{-5} \mathrm{~m}^{2}$. Perfect slip conditions over the walls are imposed for the NS stage, while ADV do not require boundary conditions, as in the former case.

The dimensionless front displacement $r_{f}\left(t^{*}\right) / r_{0}$ of the breaking column as a function of the dimensionless time $t^{*}=t \sqrt{2 g / r_{0}}$ is represented in Fig. 12, where the experimental measurements of Martin and Moyce (1952) are compared with the numerical results obtained through: (i) the present NS+ADV+BRCP approach, (ii) Edge-Tracked Interface Locator Technique (ETILT) and Moving Lagrangian Interface Remeshing Technique (MLIRT), both from Cruchaga et al. (2009), and a Least Square Finite Element Method (LSFEM) (Tang et al., 2008).

Since there are no experimental results for the dimensionless height of the top of the column, only numerical results are shown in Fig. 13, corresponding to the method introduced in the present work and the results presented in Cruchaga et al. (2009) for ETILT and MLIRT, considering the dimensionless column height $h_{c}\left(t^{*}\right) / r_{0}$ as a function of the dimensionless time $t^{*}$. All these results correspond to an aspect ratio of $r_{a}=H_{c} / W_{c}=2$, and then to similarity flows. The early stages of the collapse of the column are represented in Fig. 14.

\section{CONCLUSIONS}

The simulation of viscous and incompressible free surface flows is numerically performed by a three-stage finite element method based on the level set approximation, consisting of: a Navier-Stokes solver for the fluid flow, a transport equation for the advection of the level set function field, and a renormalization step for keeping some properties of the scalar field, in a weak-coupling paradigm. Each of the three steps is numerically computed over the same fixed finite element mesh, and can be solved through parallel computing. Particularly, the application of a continuous operator, named Bounded Renormalization with Continuous Penalization (BRCP), is introduced for two-fluid flow problems.

Appropriate values for the user-defined parameters required by the BRCP are directly proposed as (i) a function of the number of spatial dimensions for the penalizing parameter $M$, and (ii) a diffusivity $\kappa$ proportional to the square of the transition semiwidth between the limiting bounds of the level set function $\phi$. In the last case, the mesh size near the interface can be taken as a reference for proposing $\delta$.

The advection and renormalization stages are used to solve a classical test, the disk of Zalesak in Sec. 3.1, which shows the performance of the renormalization method for keeping the sharpness of the interface and the regularity of the level set function field 
obtained. The renormalization parameters are applied according to the guidelines given in Sec. 2.3, and the influence of the diffusivity $\kappa$ over the transition width $\delta$ is verified.

Two typical two and three-dimensional transient free surface problems are solved by the three-stage methodology, in Secs. 3.2 and 3.3, respectively. The results are in good agreement with experimental measurements and with the results obtained through other numerical methods taken from the literature for the early stages of the problem, showing the ability of the proposed strategy to consider large density and viscosity ratios. Furthermore, after the water impact over the walls, the present method reproduces complex topological changes, as the breaking up and merging of the free surface. The BRCP coefficients $M$ and $\kappa$ were given directly by the estimations mentioned before.

The three-stage proposal can be applied to free surface flows as well as to general twofluid flows problems, taking into account that the boundary conditions in the interface are directly solved by the two-phase level set method.

ACKNOWLEDGEMENTS

This work has received financial support from Consejo Nacional de Investigaciones Científicas y Técnicas (CONICET, Argentina, grant PIP 5271/05), Universidad Nacional del Litoral (UNL, Argentina, grant CAI+D 2009-III-4-2) and Agencia Nacional de Promoción Científica y Tecnológica (ANPCyT, Argentina, grants PICT 01141/2007, PICT 1506/2006), and was partially performed with the Free Software Foundation GNU-Project resources such as GNU/Linux OS and GNU/Octave, as well as other Open Source resources such as PETSc, MPICH, OpenDX, ParaView, LTEX and JabRef.

\section{REFERENCES}

J. Ed Akin, Tayfun E. Tezduyar, and Mehmet Ungor. Computation of flow problems with the mixed interface-tracking/interface-capturing technique (MITICT). Computers \& Fluids, 36(1):2-11, Enero 2007. URL http://www. sciencedirect.com/science/ article/B6V26-4HPD3K1-1/2/7aec2b642a2f0ae2db334e8328b8e79d.

Satish Balay, Kris Buschelman, Victor Eijkhout, William D. Gropp, Dinesh Kaushik, Matthew G. Knepley, Lois Curfman McInnes, Barry F. Smith, and Hong Zhang. PETSc users manual. ANL 95/11 - Revision 3.0.0, Argonne National Laboratory, 2008.

L. Battaglia, J. D' Elía, M. A. Storti, and N. M. Nigro. Numerical simulation of transient free surface flows using a moving mesh technique. ASME Journal of Applied Mechanics, 73(6):1017-1025, Noviembre 2006. URL http://scitation.aip.org/getabs/servlet/GetabsServlet?prog= normal\&id=JAMCAV000073000006001017000001\&idtype=cvips\&gifs= yes.

L. Battaglia, M. A. Storti, and J. D’ Elía. Stabilized free surface flows. In S. Elaskar, E. Pilotta, and G. Torres, editors, Mecánica Computacional, volume XXVI, pages 1013-1030, Cordoba, Octubre 2-5 2007. URL http://www.cimec.org.ar/ojs/.

L. Battaglia, M. A. Storti, and J. D'Elía. Bounded renormalization with continuous penalization for level set interface capturing methods. International Journal for Numerical Methods in Engineering, 2010. (accepted).

Laura Battaglia. Stabilized Finite Elements for Free Surface Flows: Tracking and Capturing of Interface. PhD thesis, Facultad de Ingeniería y Ciencias Hídricas, Universidad Nacional del Litoral., 2009. In Spanish.

Alexander N. Brooks and Thomas J. R. Hughes. Streamline upwind/Petrov-Galerkin formulations for convection dominated flows with particular emphasis on the incompressible Navier- 
Stokes equations. Computer Methods in Applied Mechanics Engineering, 32(1-3):199-259, Septiembre 1982. URL http://www. sciencedirect.com/science/article/ B6V29-47X87NJ-FY/2/4ca7fa459f21c3c49689df53c9e85b63.

S. Chippada, T. C. Jue, S. W. Joo, M. F. Wheeler, and B. Ramaswamy. Numerical simulation of free-boundary problems. International Journal of Computational Fluid Dynamics, 7(1):91-118, 1996. URL http://www.informaworld.com/10.1080/ 10618569608940754.

Marcela Cruchaga, Diego Celentano, Piotr Breitkopf, Pierre Villon, and Alain Rassineux. A surface remeshing technique for a lagrangian description of $3 \mathrm{~d}$ two-fluid flow problems. International Journal for Numerical Methods in Fluids, n/a(-):-, 2009. URL http: //dx.doi.org/10.1002/fld.2073. in press.

Marcela A. Cruchaga, Diego J. Celentano, and Tayfun E. Tezduyar. Collapse of a liquid column: numerical simulation and experimental validation. Computational Mechanics, 39:453-476, 2007.

R.N. Elias and Alvaro L. G. A. Coutinho. Stabilized edge-based finite element simulation of free-surface flows. International Journal for Numerical Methods in Fluids, 54(6-8): 965-993, Junio 2007. URL http: / / www3 . interscience.wiley . com/ journal/ 114191654 /abstract.

R.N. Elias, Marcos A. D. Martins, and Alvaro L. G. A. Coutinho. Simple finite element-based computation of distance functions in unstructured grids. International Journal for Numerical Methods in Engineering, 72(9):1095-1110, Noviembre 2007. URL http://www3.interscience.wiley.com/cgi-bin/abstract/ 114250638 /ABSTRACT ?CRETRY=1\&SRETRY=0.

Joao Paulo Gois, Anderson Nakano, Luis Gustavo Nonato, and Gustavo C. Buscaglia. Front tracking with moving-least-squares surfaces. Journal of Computational Physics, 227(22): 9643-9669, Noviembre 2008. URL http: //www. sciencedirect. com/science/ article/B6WHY-4T5CHOY-1/2/ee 4 c 91 ef 6081 ba 0 f3d3942 facle 50 fd4.

C. W. Hirt and B. D. Nichols. Volume of fluid (VOF) method for the dynamics of free boundaries. Journal of Computational Physics, 39(1):201-225, Enero 1981.

Antonio Huerta and Wing Kam Liu. Viscous flow with large free surface motion. Computer Methods in Applied Mechanics and Engineering, 69:277-324, 1988.

Thomas J. R. Hughes, Wing Kam Liu, and Thomas K. Zimmermann. LagrangianEulerian finite element formulation for incompressible viscous flows. Computer Methods in Applied Mechanics and Engineering, 29(3):329-349, Diciembre 1981. URL http://www.sciencedirect.com/science/article/ B6V29-47X87MD-F8/1/9c2b5f4ae597f644298c2eadf346e3c5.

Shu-Ren Hysing. Numerical Simulation of Immiscible Fluids with FEM Level Set Techniques. PhD thesis, Dortmund - Germany, 2007.

S. R. Idelsohn, E. Oñate, and F. Del Pin. The Particle Finite Element Method: a powerful tool to solve incompressible flows with free-surfaces and breaking waves. International Journal for Numerical Methods in Engineering, 61(7):964-984, 2004.

Shunsuke Kurioka and David R. Dowling. Numerical simulation of free surface flows with the level set method using an extremely high-order accuracy weno advection scheme. International Journal of Computational Fluid Dynamics, 23(3):233-243, 2009. URL http: //www. informaworld.com/10.1080/10618560902776786.

Linux. The linux documentation project. http://www.gnu.org/, 2010. URL http://www. gnu . org/. URL http://www.gnu.org/. 
Emilie Marchandise and Jean-Francois Remacle. A stabilized finite element method using a discontinuous level set approach for solving two phase incompressible flows. Journal of Computational Physics, 219(2):780-800, 2006. URL (http: //www. sciencedirect. com/science/article/B6WHY-4K66F 9X-1/2/ b106f78d1d4e08dc091c94869cb1b789).

J. C. Martin and W. J. Moyce. An experimental study of the collapse of liquid columns on a rigid horizontal plane. Philosophical Transactions of the Royal Society of London. Series A, Mathematical and Physical Sciences, 244(882):312324, Marzo 1952. URL http://journals.royalsociety.org/content/ x58vt522r1326111/?p=a15f021e176b40a89b7423ce8cc14b72\&pi=0.

MPI. Message passing interface, 2009. URL http://www.mpi-forum.org. http://www.mpi-forum.org.

Fernando Mut, Gustavo C. Buscaglia, and Enzo A. Dari. New mass-conserving algorithm for level set redistancing on unstructured meshes. Journal of Applied Mechanics, 73(6):1011-1016, Noviembre 2006. URL http://asmedl. aip.org/getabs/servlet/GetabsServlet?prog=normal\&id= JAMCAV000073000006001011000001\&idtype=cvips\&gifs=Yes.

Ellin Olsson and Gunilla Kreiss. A conservative level set method for two phase flow. Journal of Computational Physics, 210(1):225-246, Noviembre 2005. URL http: //www. sciencedirect.com/science/article/B6WHY-4GFNGDW-4/ 1/c6e8193acd9920efe4e3954e28cd1379.

Ellin Olsson, Gunilla Kreiss, and Sara Zahedi. A conservative level set method for two phase flow II. Journal of Computational Physics, 225(1):785-807, Julio 2007. URL http://www.sciencedirect.com/science/article/ B6WHY-4MYMG2M-1/1/739bd766e696bd913f335cb19e078d86.

Stanley Osher and James A. Sethian. Fronts propagating with curvature-dependent speed: algorithms based on Hamilton-Jacobi formulations. Journal of Computational Physics, 79 (1):12-49, Noviembre 1988. URL http://www. sciencedirect.com/science/ article/B6WHY-4DD1R90-2/1/21c38ec71e0d62474b52338614e73aff.

PETSc-FEM. A general purpose, parallel, multi-physics FEM program, 2009. URL http://www.cimec.org.ar/petscfem. GNU General Public License (GPL), http://www.cimec.org.ar/petscfem.

Sylvaine Rabier and Marc Medale. Computation of free surface flows with a projection FEM in a moving mesh framework. Computer Methods in Applied Mechanics and Engineering, 192(41-42):4703-4721, Octubre 2003. URL http: //www. sciencedirect.com/science/article/B6V29-49FR9V5-1/ 1/79ade753674f0aa2fd437e265fcb1642.

Ruben Scardovelli and Stéphane Zaleski. Direct numerical simulation of free-surface and interfacial flow. Annual Reviews in Fluid Mechanics, 31:567-603, 1999.

J. A. Sethian. A Fast Marching Level Set Method for Monotonically Advancing Fronts. National Academy of Sciences, 1995.

W. Shyy, H. S. Udaykumar, M. M. Rao, and R. W. Smith. Computational Fluid Dynamics with Moving Boundaries. Taylor and Francis, 1996.

V.E. Sonzogni, A. Yommi, N.M. Nigro, and M.A. Storti. A parallel finite element program on a Beowulf Cluster. Advances in Engineering Software, 33:427-443, 2002.

M. A. Storti, N. M Nigro, R. R. Paz, and L. D. Dalcín. Dynamic boundary conditions in computational fluid dynamics. Comp. Meth. in Appl. Mech. and Engr., 197(13-16): 
1219-1232, 2008. URL http://www. sciencedirect.com/science/article/ B6V29-4R41HX0-1/2/c4ad5a02d8c46b7050b30345df05f890. Elsevier, Amsterdam, The Netherlands.

Mark Sussman, Ann S. Almgren, John B. Bell, Phillip Colella, Louis H. Howell, and Michael L. Welcome. An adaptive level set approach for incompressible two-phase flows. Journal of Computational Physics, 148(1):81-124, Enero 1999. URL http://www.sciencedirect.com/science/article/ B6WHY-45K197N-5/1/367632e72cc0fbf9481df0e8a6563cle.

Mark Sussman and Peter Smereka. Axisymmetric free boundary problems. Journal of Fluid Mechanics, 341:269-294, 1997.

Bo Tang, Jun-Feng Li, and Tian-Shu Wang. Viscous flow with free surface motion by least square finite element method. Applied Mathematics and Mechanics, 29(7):943-952, Julio 2008. URL http://www. springerlink. com/content/tt8w2w2838874v47/.

T. E. Tezduyar, S. Mittal, S. E. Ray, and R. Shih. Incompressible flow computations with stabilized bilinear and linear-equal-order interpolation velocity-pressure elements. Computer Methods in Applied Mechanics and Engineering, 95(2):221-242, Marzo 1992. URL http://www.sciencedirect.com/science/article/ B6V29-47YJHF 8-N/2/26eeb53a02cb6831f97aeef29f $9 b a 584$.

Tayfun E. Tezduyar. Interface-tracking and interface-capturing techniques for finite element computation of moving boundaries and interfaces. Comp. Meth. in Appl. Mech. and Engr., 195(23-24):2983-3000, 2006.

Steven T. Zalesak. Fully multidimensional flux-corrected transport algorithms for fluids. Journal of Computational Physics, 31(3):335-362, Junio 1979. URL http: //www. sciencedirect.com/science/article/B6WHY-4DD1XT7-1R9/1/ 01a0aa89abd6872e4b605a005a28c991. 


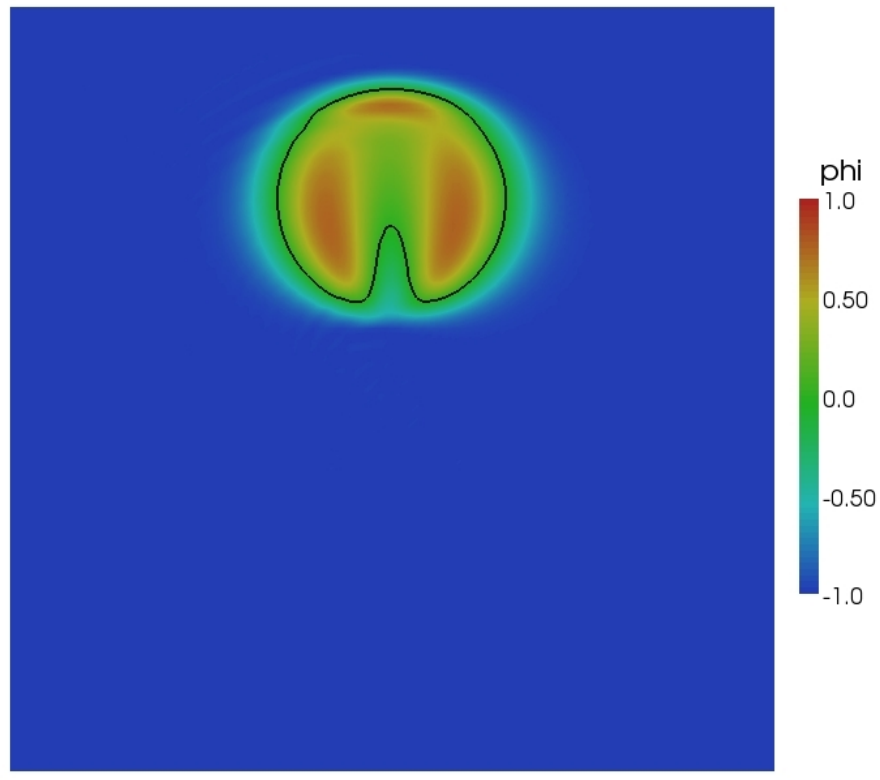

(a) ADV

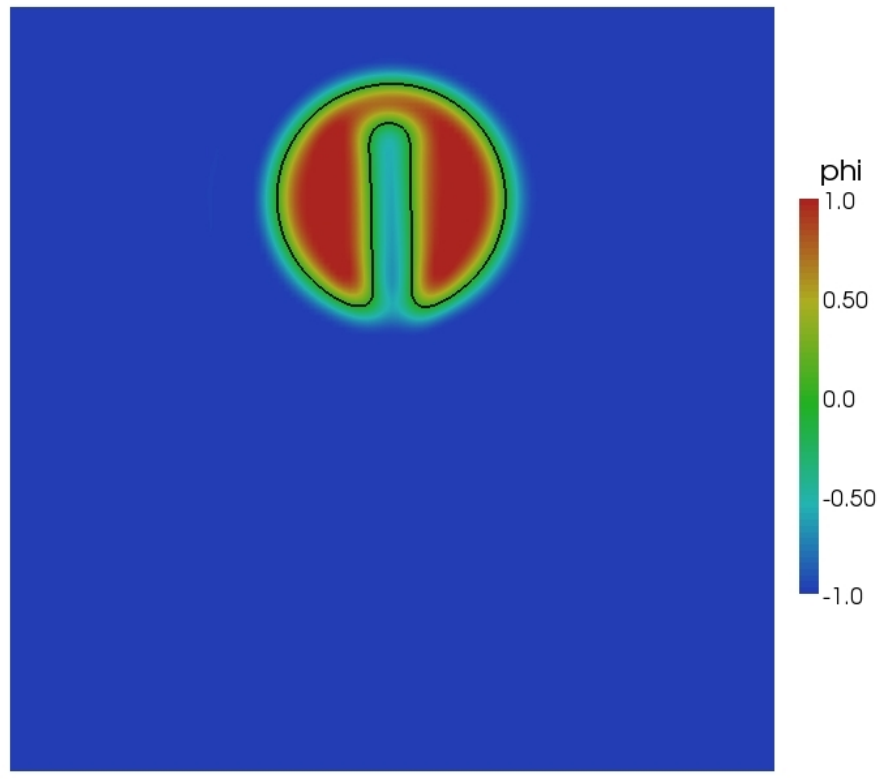

(b) $\mathrm{ADV}+\mathrm{BRCP}(\mathrm{A})$

Figure 5: Level set function field at $t=1 \mathrm{~s}$ for the case of the notched disk. 


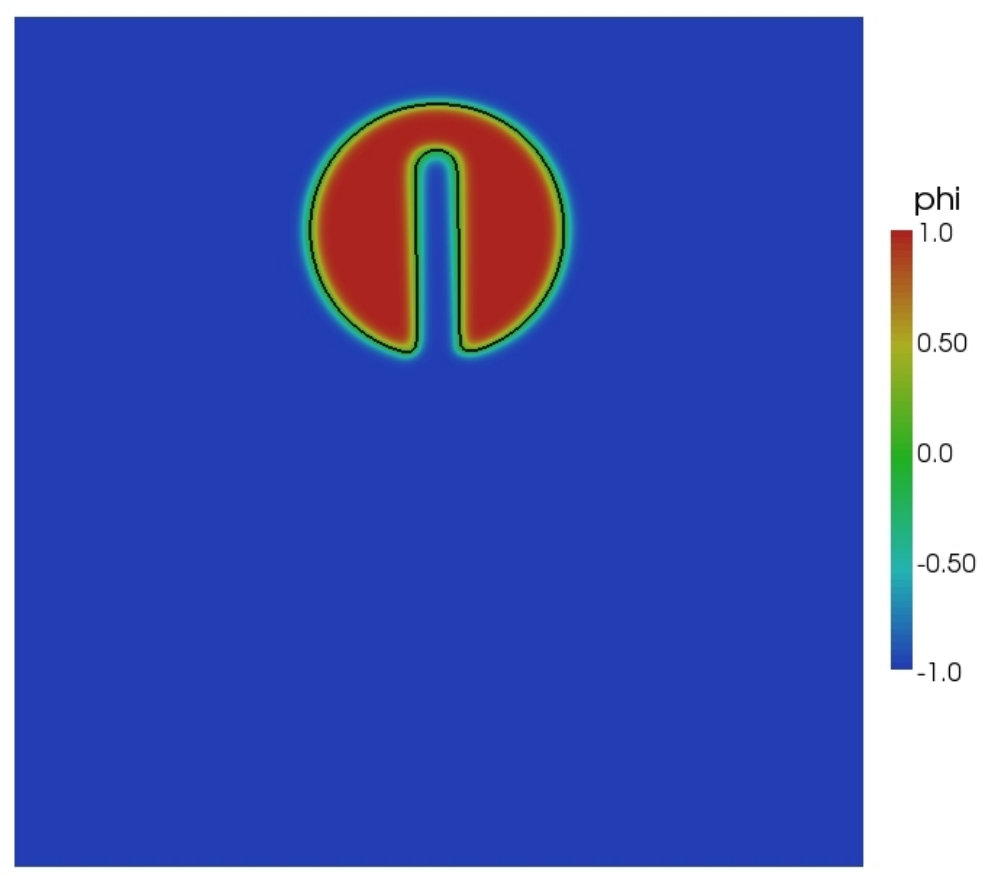

(a) LS function field

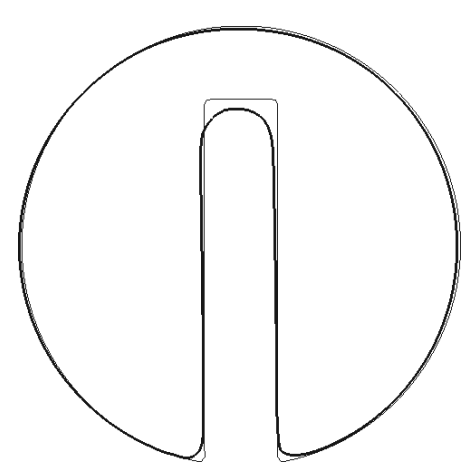

(b) initial (thin) and final (thick) $\phi=0$ profiles

Figure 6: Results of the analysis for the rotating disk with the refined mesh. 


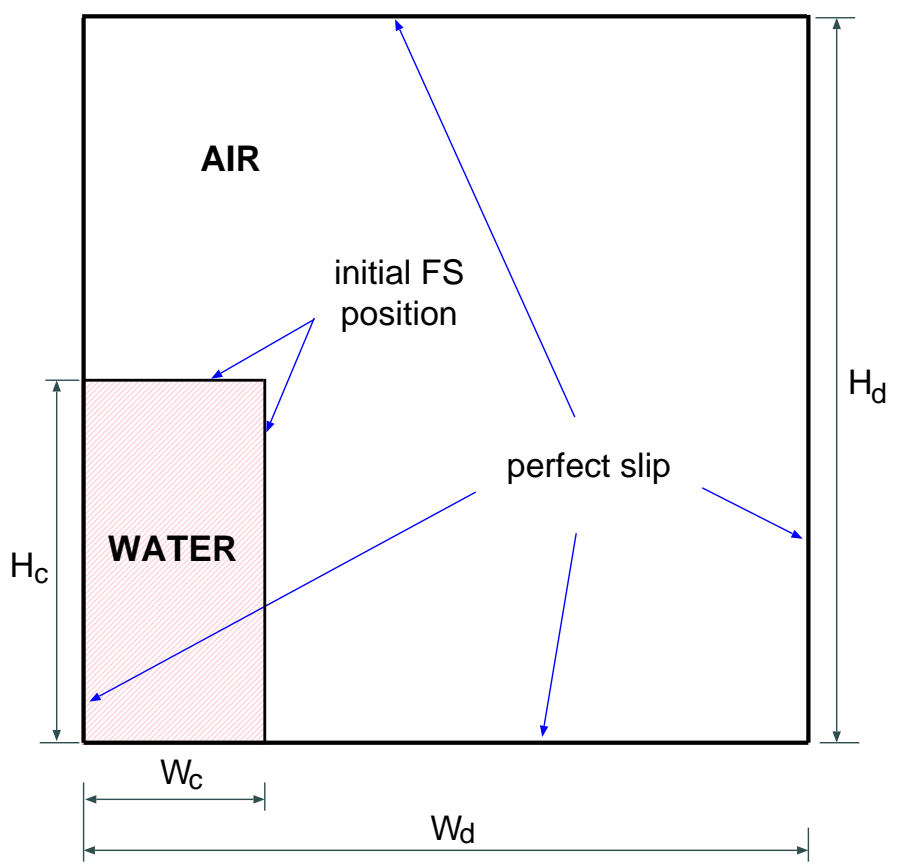

Figure 7: Geometry and boundary conditions for the collapse of a water column example in 2D.

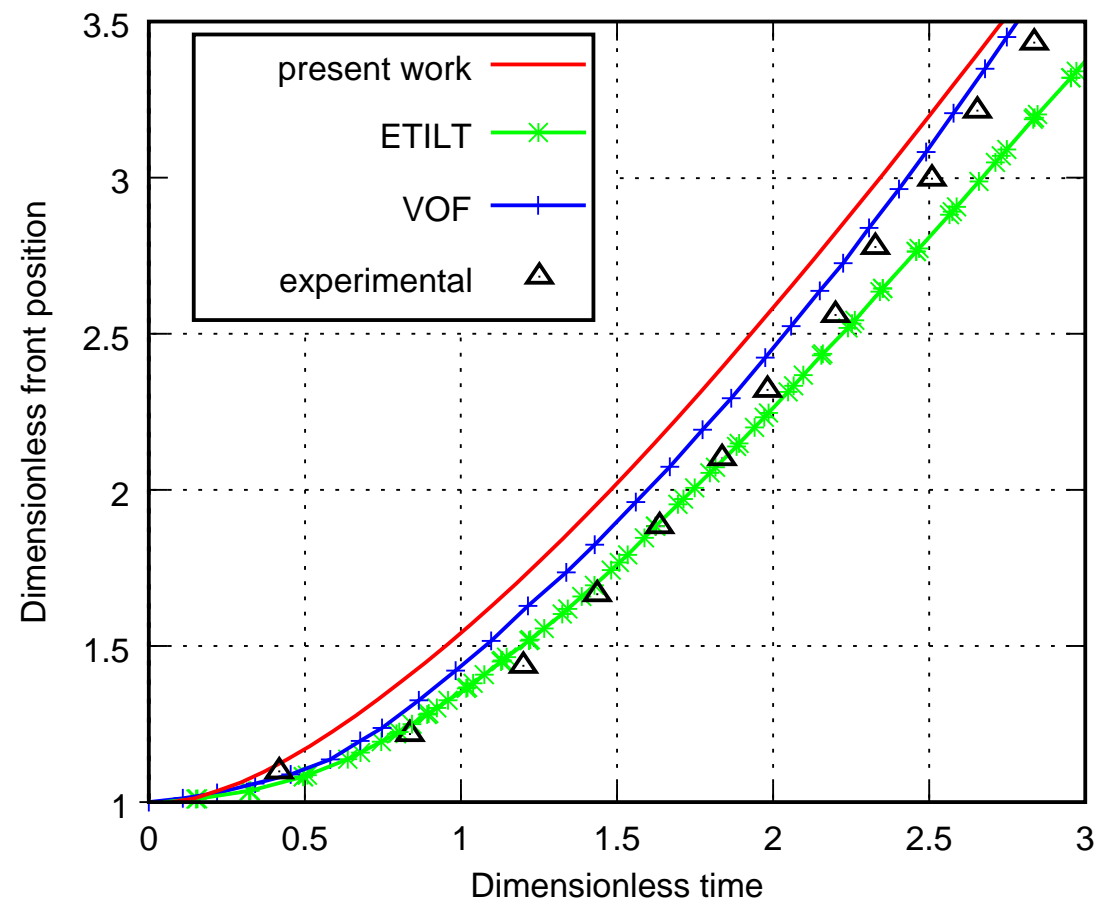

Figure 8: Dimensionless front position $x_{f}\left(t^{*}\right) / W_{c}$ versus dimensionless time $t^{*}=t \sqrt{2 g / W_{c}}$ for the 2D dambreak problem: numerical results and experimental data. 


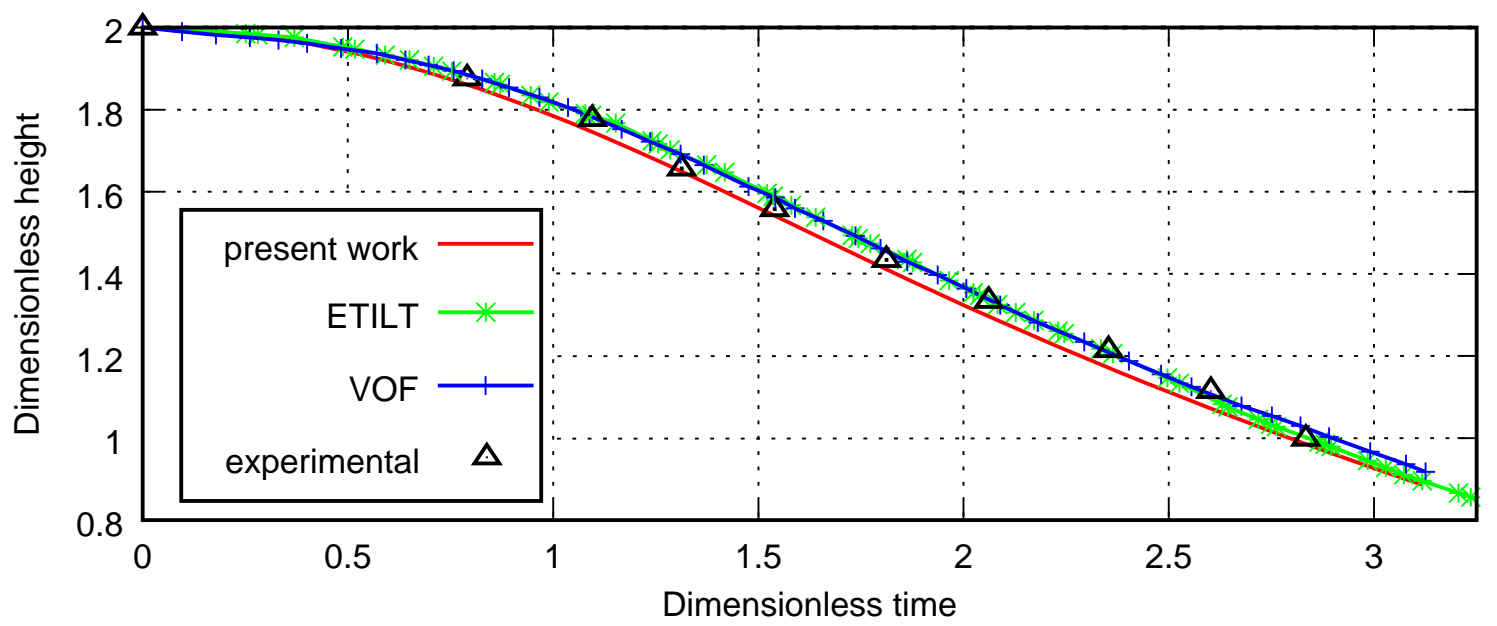

Figure 9: Dimensionless column height $h_{c}\left(t^{*}\right) / W_{c}$ versus dimensionless time $t^{*}=t \sqrt{2 g / W_{c}}$ for the problem of the 2D dam-break problem: numerical results and experimental data. 

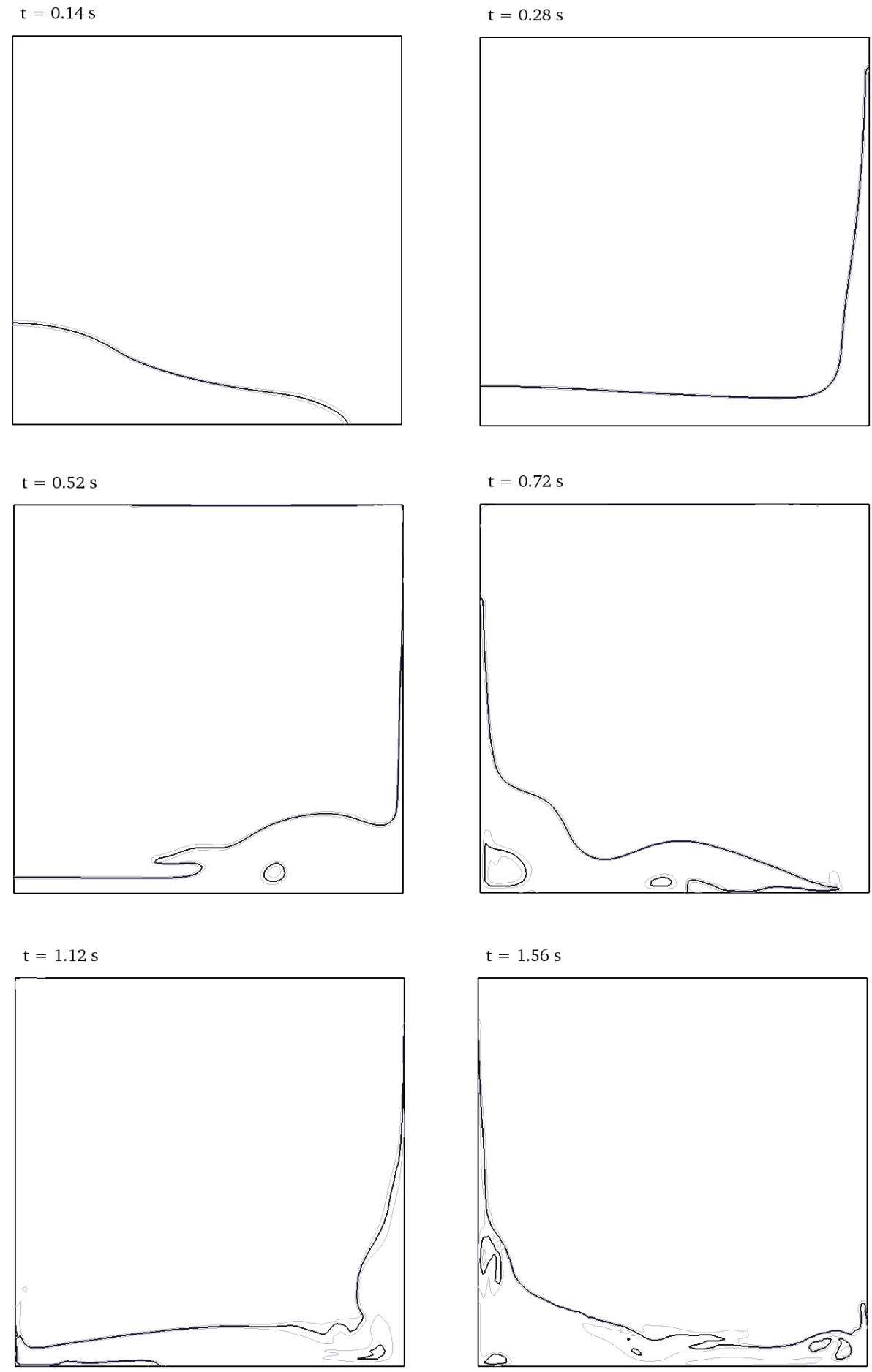

Figure 10: Free surface positions for several instants in the 2D dam-break problem. 

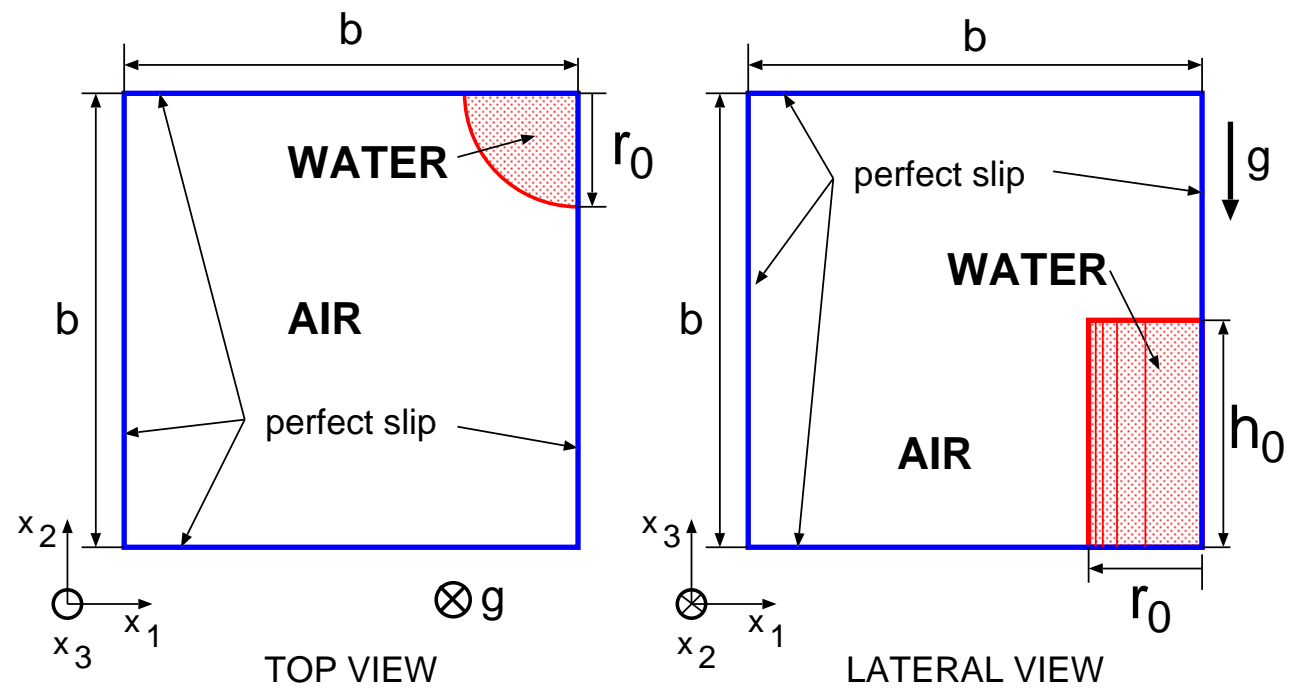

Figure 11: Geometry for the problem of the collapse of a cylindrical water column in 3D.

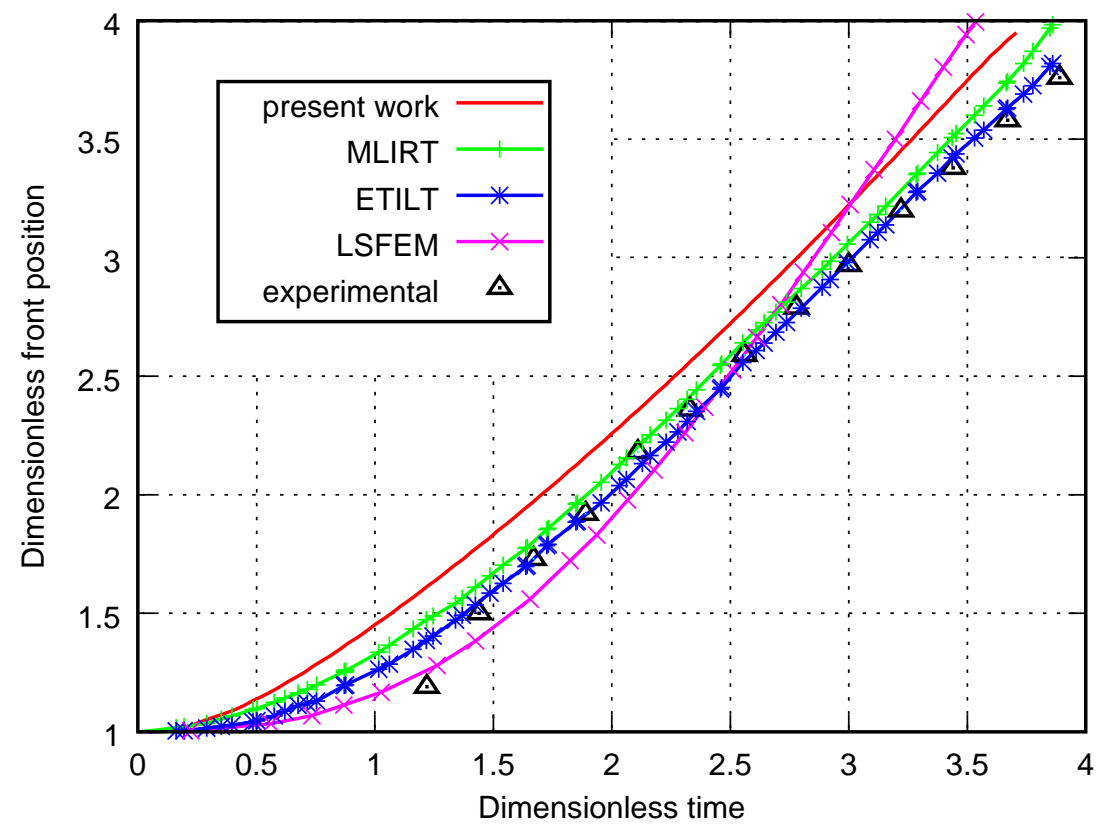

Figure 12: Dimensionless front position $r_{f}\left(t^{*}\right) / r_{0}$ versus dimensionless time $t^{*}=t \sqrt{2 g / r_{0}}$ for the problem of the collapse of a cylindrical water column in 3D: numerical results and experimental data. 


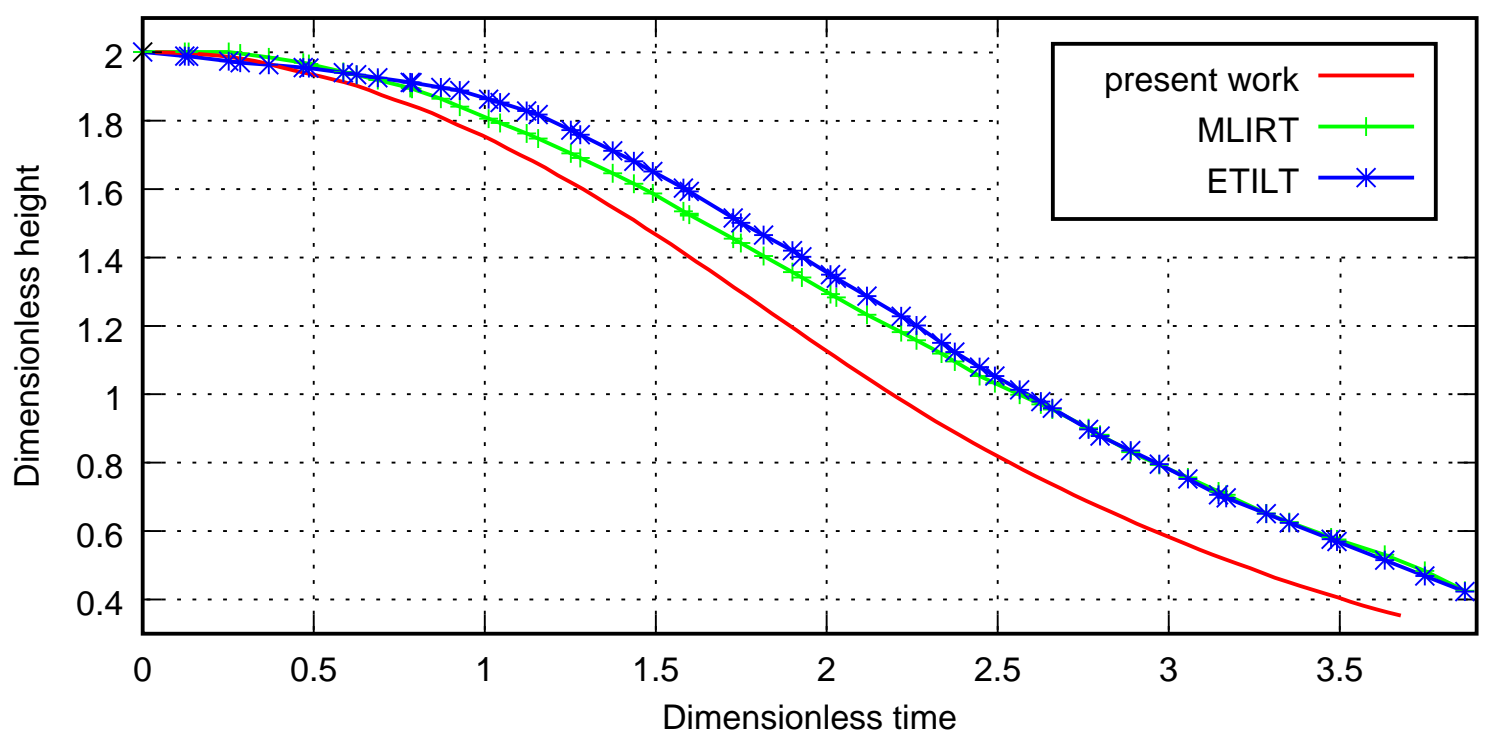

Figure 13: Dimensionless column height $h_{c}\left(t^{*}\right) / r_{0}$ versus dimensionless time $t^{*}=t \sqrt{2 g / r_{0}}$ for the problem of the collapse of a cylindrical water column in 3D: numerical results. 

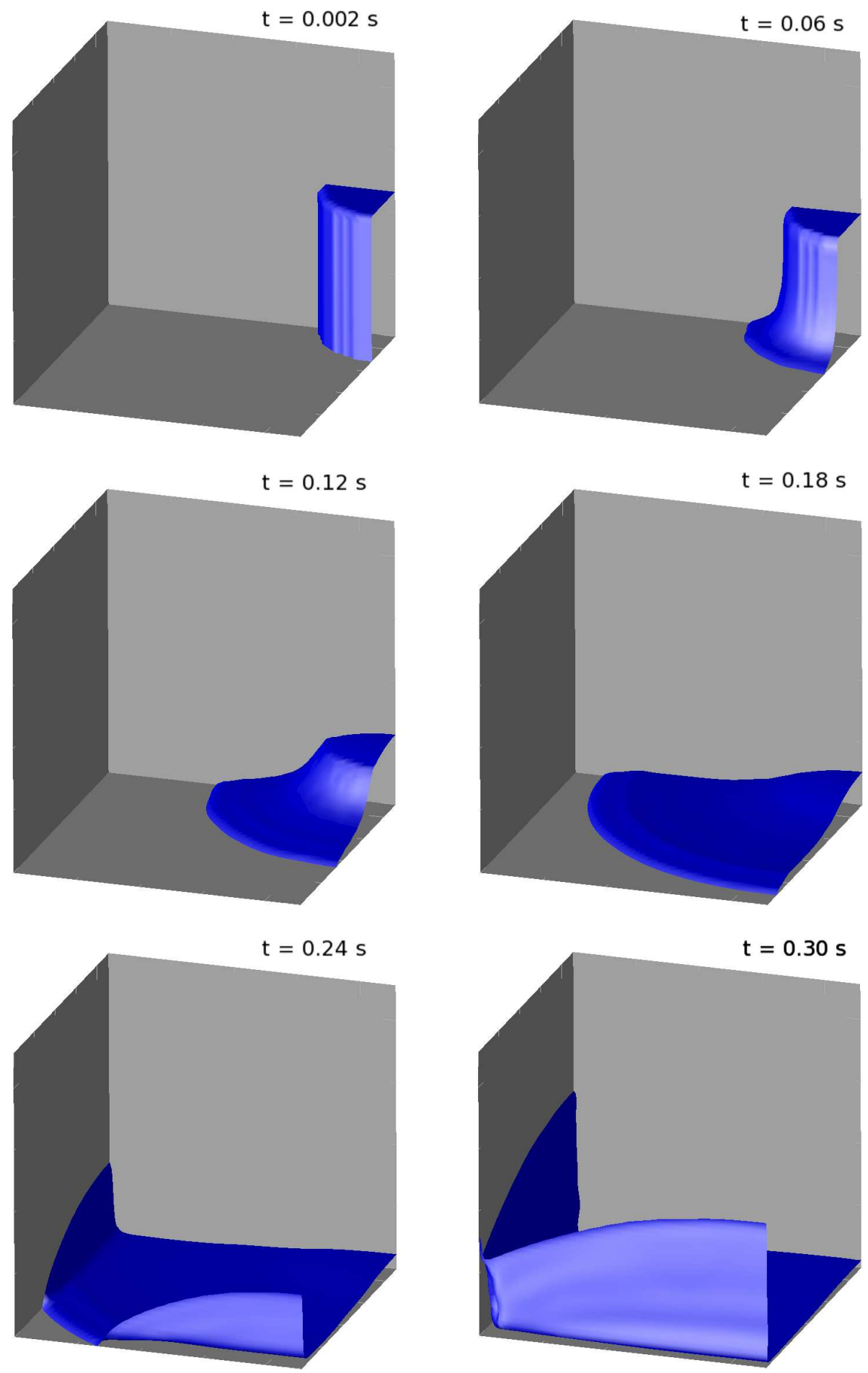

Figure 14: Initial stages for the problem of the collapse of a cylindrical water column in 3D. 